Monatsschrift f. Geburtshülfe u. Gynäkologie 1930;84:I-IV

\title{
Contents, Vol. 84, 1930
}

Inhaltsverzeichnis.

Originalarbeiten. $\quad \mathrm{S} \theta \mathrm{it} \theta$

Börner, Rudolf, Mechanik der ,,Myomwanderung” im graviden Uterus 321

- $\quad$ siehe Küstner, Heinz.

Brock, James, Vererbungslehre und Geburtshilfe

Conill, Victor, Reines Scheidenmyxom

Gidalewitsch, N. A., Das Puerperium nach Vielfruehtgravidität. . . 261

Gisbertz, H., Zur Morphologie der Glykogenablagerung im Vaginalepithel 24

He gar, siehe Sellheim, H., Tauffer, W. uvd Martin, A.

Heim, K., Die örtlichen Betäubungsverfahren in der Frauenheilkundc 45

Hoe de, Karl, Über Bezielmngen zwischen Psoriasis und Ovarialfunktion 346

Hoffmann, Erich, Kongenitale Syphilis (Lues innata) 122

Janáki, Julius, Bestrahlungen mit kleinen Dosen bei Entzündungen

der Adnexe 142

Istel, Franz, Echte Bauchhöhlenschwangerschaft

366

- $\quad$ Uterusruptur in der Schnittnarbe nach Sectio caesarea

Itzkin, Simon, Nochmals zur Frage der Toleranz des Uterus duplex

gravidus bei wiederholter Ausschabung der nichtgraviden Hälfte . 249 Kakuschkin, N.,

Transplantation des Eileiters von einem Weibe zum

anderen 394

Karakalos, Andreas, Uber einen seltenen Fall schwerer Schwanger-

schaftstachykardie 95

Kayser,Marie-Elise, Frauenmilch-Sammelstelle (F.M.S.) 293

Kleiner, B., Zur Frage der Bauchspaltung beim Neugeborenen. Zwei

eigene Fälle 281

Klenitzky, Jacob, ísolierte Scheidengewölbeverletzungen sub coitu 40J Küstner, Heinz, und

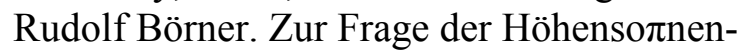

bestrahlung der laktierenden Mamma275

Lehfeldt, H., siehe Sakheim, J.

Mahnert, A., und H. Siegmund, Störungen des Zyklus durch Hyper-

hormonisierung $\quad 91$

Malfatti, Josef, Zur Polyomyositis septiea 373

Martin, A., Persönliche Erinnerungen an Alfred Hegar 22

Martin, Ed., und Vierkotten, Die angeborene Syphilis 128

Mashbitz, A.M., Die puerperale Thrombophlebitis 31

Mathias, Ernst, Ein kasuistischer Beitrag zur Kollis'ion zwischen

Berufsgeheimnis und Anzeigepnicht 406

Naujoks, H.. Zur Bewertung des Avertins (Avertintodesfall, Avertin-

exanthem, Avertin bei Eklampsie) 97 
Reichelt, Hertha, Ergebnisse der Abortbehandlung in den letzten

10 Jahren 357

Rieck, A., Kann die serosaepitheliale Theorie über die Entstehung der

endometrioiden Heterotopien noch aufrecht erhalten werden? . . 336 Sakheim, J., und Lehfeldt, H., Klímakteríum und Blutbild. Ein Beitrag zur differentialdiagnostischen Wertung der relativen Lyrnpho-zytose und ein Erklärungsversuch der günstigen Wirkung von

Aschners ,entgiftender” Therapie im Klimakterium 241

S e i t z, L u d w i g, Über den Einfluß der i $\pi$ neren Sekretion auf Entwicklung

und Erkrankung der weiblichen Sexualsphäre und über Hormon-

therapie gynäkologischer Störungen 79

IV Inhaltsverzeichnis.

Seitfr Sellheim, Hugo,Hegar als Geburtshelfer. Eine Erinnerung zu seinem

100. Geburtstage 3

- Über Verschiebung von Fibromen im Uterus

Siegmund, H., siehe Mahnert, A.

Tauffer, W. , Hegars Wirken im Geiste Semmelweis". Ein Beitrag zur

Zeitgeschichte 8

Vierkotten, siehe Martin, Ed.

Willbrand,L., Über die Erfolge der Eileiterdurchblasung bei Sterilität 63

Wolf, Herbert, Interstitielle Tubenschwangerschaften Ill

Ernst Bauer $\uparrow 468$

Wilhelm Zangemeister $\dagger \quad 470$

Sammelberichte.

Schneider, G. H., Der Röntgenkater und seine Bekämpfung .... 4091

Berichte über ausländische Literatur.

Übersicht über die im Jahre 1928 veröffentlichten amerikanischen Arbeiten

auf gynäkologisch-geburtshilf lichem Gebiete. Von Dr. ArthurStein-

New York 145

Die italienische geburtshilflich-gynäkologisehe Literatur (1929, I. Sem.).

Von Privatdozent Dr. Sebastiano di Francesco-Mailand . . . 154

Vereîns- und Literaturbeîlage.

Original-Sitzungsberichte aus geburtshilflich-gynäkologischen Gesellschafter:

Gesellschaft für Geburtshilfe und Gynäkologie zu Berlin. Sitzungen vom

25. X. und 8. XI. $1929 \quad 180$

Sitzung vom 22. XL $1929 \quad 309$

Sitzungen vom 13. XII. 29 und 10. I. $30 \quad 424$

Gynäkologische Gesellschaft zu Breslau. Sitzung vom 19. XI. 1929 . 190

- $\quad$ Sitzung vom 21. I. $1930 \quad 434$

Nordwestdeutsche Gesellschaft für Gynäkologie. Sitzung vom 5. X. 1929

in Hannover 69

Indikationen und Teclinik der Sterilisierung der Frau. ßeferate des VI. Kongresses der

Frauenärzte und Geburtshelfer des französischen Sprachgebietes in Brüssel vom 3. bis 5. X.

1029. Von G. H. Schneider-

Brandenburg 76

Niederrheinisch-westfälische Gesellschaft für Gynäkologie und Geburts

hilfe. 91. Sitzung zu Düsseldorf am 16. XI. 1929 
Mittelrheinische Gesellschaft für Geburtshilfe und Gynäkologie. 80. Sitzung zu Frankfurt a. M. am 27. X. 1929224

- $\quad$ Sitzung vom 8. XII. 1929 zu Frankfurt a. M 445

Geburtshilflich-gynäkologisehe Gesellschaft in Wien. Sitzung vom

5. XI. 1929223

Sitzung vom 10. XII. 1929313

Sitzung vom 14. I. $1930 \quad 462$

Buchbesprechungen 78, 238, 316, 464

Personalien und Tagesnachrichten $78,239,240,472$ 